\title{
Non-Nehari Manifold Method for Fractional p-Laplacian Equation with a Sign-Changing Nonlinearity
}

\author{
Huxiao Luo, ${ }^{1}$ Shengjun Li $\left(\mathbb{D},{ }^{2,3}\right.$ and Wenfeng $\mathrm{He}^{2}$ \\ ${ }^{1}$ Department of Mathematics, Zhejiang Normal University, Jinhua, Zhejiang 321004, China \\ ${ }^{2}$ College of Information Sciences and Technology, Hainan University, Haikou 570228, China \\ ${ }^{3}$ School of Mathematics and Statistics, Central South University, Changsha, Hunan 410083, China \\ Correspondence should be addressed to Shengjun Li; shjli626@126.com
}

Received 15 April 2018; Accepted 10 June 2018; Published 18 July 2018

Academic Editor: Xinguang Zhang

Copyright (C) 2018 Huxiao Luo et al. This is an open access article distributed under the Creative Commons Attribution License, which permits unrestricted use, distribution, and reproduction in any medium, provided the original work is properly cited.

We consider the following fractional p-Laplacian equation: $(-\Delta)_{p}^{\alpha} u+V(x)|u|^{p-2} u=f(x, u)-\Gamma(x)|u|^{q-2} u, x \in \mathbb{R}^{N}$, where $N \geq 2$, $p_{\alpha}^{*}>q>p \geq 2, \alpha \in(0,1),(-\Delta)_{p}^{\alpha}$ is the fractional $p$-Laplacian, and $\Gamma \in L^{\infty}\left(\mathbb{R}^{N}\right)$ and $\Gamma(x) \geq 0$ for a.e. $x \in \mathbb{R}^{N} . f$ has the subcritical growth but higher than $\Gamma(x)|u|^{q-2} u$; however, the nonlinearity $f(x, u)-\Gamma(x)|u|^{q-2} u$ may change sign. If $V$ is coercive, we investigate the existence of ground state solutions for p-Laplacian equation.

\section{Introduction}

Consider the following nonlinear Schrödinger equation with fractional $p$-Laplacian:

$$
\begin{array}{r}
(-\Delta)_{p}^{\alpha} u+V(x)|u|^{p-2} u=f(x, u)-\Gamma(x)|u|^{q-2} u, \\
x \in \mathbb{R}^{N},
\end{array}
$$

where $N \geq 2, p_{\alpha}^{*}>q>p \geq 2, \alpha \in(0,1)$, and $(-\Delta)_{p}^{\alpha}$ is the fractional $p$-Laplacian. $V(x), \Gamma(x)$, and $f(x, u): \mathbb{R}^{N} \times \mathbb{R} \longrightarrow$ $\mathbb{R}$ satisfy the following assumptions:

$(V) V \in C\left(\mathbb{R}^{N}, \mathbb{R}\right), V_{0}:=\inf _{x \in \mathbb{R}^{N}} V(x)>0$, there exists a constant $d_{0}>0$ such that

$\lim _{|y| \rightarrow \infty} \operatorname{meas}\left\{x \in \mathbb{R}^{N}:|x-y| \leq d_{0}, V(x) \leq M\right\}=0$,

$$
\forall M>0,
$$

where meas $(\cdot)$ denotes the Lebesgue measure in $\mathbb{R}^{N}$;

( Г ) $\Gamma \in L^{\infty}\left(\mathbb{R}^{N}\right), \Gamma(x) \geq 0$ for a.e. $x \in \mathbb{R}^{N}$; $\left(f_{1}\right) f(x, t): \mathbb{R}^{N} \times \mathbb{R} \longrightarrow \mathbb{R}$ is measurable, continuous in $t \in \mathbb{R}$ for a.e. $x \in \mathbb{R}^{N}$ and there are $C>0$ and $2 \leq p<q<r<p_{\alpha}^{*}$ such that

$$
|f(x, t)| \leq C\left(1+|t|^{r-1}\right) \quad \text { for all } t \in \mathbb{R}, x \in \mathbb{R}^{N},
$$

where $p_{\alpha}^{*}=N p /(N-p \alpha)$;

$\left(f_{2}\right) f(x, t)=o\left(|t|^{p-1}\right)$ as $|t| \longrightarrow 0$ uniformly in $x \in \mathbb{R}^{N}$;

$\left(f_{3}\right) F(x, t) /|t|^{q} \longrightarrow \infty$ uniformly in $x$ as $|t| \longrightarrow \infty$, where $F(x, t)=\int_{0}^{t} f(x, \tau) d \tau ;$

$\left(f_{4}\right) t \longmapsto f(x, t) /|t|^{q-1}$ is nondecreasing on $(-\infty, 0) \cup$ $(0, \infty)$.

When $p=2$, (1) arises in the study of the nonlinear Fractional Schrödinger equation

$$
(-\Delta)^{\alpha} u+V(x) u=f(x, u)-\Gamma(x)|u|^{q-2} u,
$$

$$
x \in \mathbb{R}^{N} .
$$

Problems with this type have occurred in many different fields, such as continuum mechanics, phase transition phenomena, population dynamics, and game theory, as they are 
the typical outcome of stochastically stabilization of Lévy processes; see [1-4].

When $\alpha=1$ and $\Gamma=0$, (4) reduces to be the nonlinear Schrödinger equation

$$
-\Delta u+V(x) u=f(x, u), \quad x \in \mathbb{R}^{N} .
$$

Using the Nehari-type monotonicity condition, Szulkin and Weth [5] obtained the existence of ground state solutions for (5). But in this paper, the Nehari manifold is usually not smooth and the Nehari-type monotonicity condition for the nonlinearity is not satisfied; then the Nehari manifold method is invalid. In this paper, we are aimed to obtain ground state solutions for (1) by the so-called non-Nehari manifold method, which is established by Tang [6, 7]. Unlike the Nehari manifold method, the main idea of our approach lies on finding a minimizing sequence for the energy functional outside the Nehari manifold by using the diagonal method.

Now, we are ready to state the main result of this paper.

Theorem 1. Suppose that $(V),(\Gamma)$ and $\left(f_{1}\right)-\left(f_{4}\right)$ hold. Then (1) has a nontrivial ground state solution.

\section{Preliminaries}

In the paper, we will denote $o_{n}(1)$ by the infinitesimal as $n \longrightarrow$ $+\infty$. For the sake of simplicity, the norm of the space $L^{p}\left(\mathbb{R}^{N}\right)$ will be denoted by $\|\cdot\|_{p}$, and integrals over the whole $\mathbb{R}^{N}$ will be written $\int$.

We define the Gagliardo seminorm by

$$
[u]_{\alpha, p}=\left(\int \frac{|u(x)-u(y)|^{p}}{|x-y|^{N+\alpha p}} d x d y\right)^{1 / p},
$$

where $u: \mathbb{R}^{N} \longrightarrow \mathbb{R}$ is a measurable function. Then fractional Sobolev space $W^{\alpha, p}\left(\mathbb{R}^{N}\right)$ is given by

$$
\begin{aligned}
& W^{\alpha, p}\left(\mathbb{R}^{N}\right) \\
& =\left\{u \in L^{p}\left(\mathbb{R}^{N}\right): u \text { is measurable and }[u]_{\alpha, p}<\infty\right\}
\end{aligned}
$$

endowed with the norm

$$
\|u\|_{\alpha, p}=\left([u]_{\alpha, p}^{p}+\|u\|_{p}^{p}\right)^{1 / p}
$$

For the basic properties of fractional Sobolev spaces, we refer the interested reader to [8]. By condition $(V)$, we define the fractional Sobolev space with potential $V(x)$ by

$$
E:=\left\{u \in W^{\alpha, p}: \int V(x)|u|^{p} d x<\infty\right\}
$$

endowed with the norm

$$
\|u\|:=\left([u]_{\alpha, p}^{p}+\int V(x)|u|^{p} d x\right)^{1 / p} .
$$

The energy functional $J: E \longrightarrow \mathbb{R}$ defined by

$$
\begin{aligned}
J(u)= & \frac{1}{p} \int \frac{|u(x)-u(y)|^{p}}{|x-y|^{N+\alpha p}} d x d y \\
& +\frac{1}{p} \int V(x)|u(x)|^{p} d x \\
& -\int\left(F(x, u)-\frac{1}{q} \Gamma(x)|u|^{q}\right) d x .
\end{aligned}
$$

Under our hypotheses, $J$ is well defined on $E$. It is well known that $J \in C^{1}(E, \mathbb{R})$, and its derivative is given by

$$
\begin{aligned}
& \left\langle J^{\prime}(u), v\right\rangle \\
& =\int_{\mathbb{R}^{2 N}} \frac{|u(x)-u(y)|^{p-2}(u(x)-u(y))(v(x)-v(y))}{|x-y|^{N+\alpha p}} d x d y \\
& \quad+\int_{\mathbb{R}^{N}} V(x)|u|^{p-2} u v d x \\
& \quad-\int\left(f(x, u) v-\Gamma(x)|u|^{q-2} u v\right) d x,
\end{aligned}
$$

for $u, v \in E$. It is standard to verify that the weak solutions of (1) correspond to the critical points of $J$. Now, we review the main embedding result for the space $E$.

Lemma $2([9$, Lemma 1$])$. Under assumption $(V)$, the embedding $E \hookrightarrow L^{r}\left(\mathbb{R}^{N}\right)$ is compact for any $r \in\left[p, p_{\alpha}^{*}\right)$.

In the following lemma, we will show that $J$ has Mountain Pass geometric structure.

Lemma 3. Suppose that $(V),(\Gamma)$, and $\left(f_{1}\right)-\left(f_{4}\right)$ hold.

(i) There is $\delta_{0}>0$ such that $\rho_{0}:=\inf _{\|u\|=\delta_{0}} J(u)>J(0)=0$.

(ii) For any $u \neq 0$, there exists $t>0$ such that $J(t u)<0$.

Proof. (i) By $\left(f_{1}\right)$ and $\left(f_{2}\right)$, we have

$$
\begin{aligned}
& |f(x, u)| \leq \varepsilon|u|^{p-1}+C_{\varepsilon}|u|^{r-1}, \\
& |F(x, u)| \leq \frac{\varepsilon}{p}|u|^{p}+\frac{C_{\varepsilon}}{r}|u|^{r} .
\end{aligned}
$$

By $E \hookrightarrow L^{s}\left(\mathbb{R}^{N}\right)$ for $s \in\left[p, p_{\alpha}^{*}\right)$ and (13), we have

$$
\begin{aligned}
J(u) & =\frac{1}{p}\|u\|^{p}-\int F(x, u) d x+\frac{1}{q} \int \Gamma(x)|u|^{q} d x \\
& \geq \frac{1}{p}\|u\|^{p}-\varepsilon C_{1}\|u\|^{p}-C_{\varepsilon} C_{2}\|u\|^{r} .
\end{aligned}
$$

By the arbitrariness of $\varepsilon$ and $p<r$, we get the conclusion.

(ii) Fix $u \neq 0$; by $\left(f_{3}\right)$, we have

$$
\begin{aligned}
\frac{J(t u)}{t^{q}}= & \frac{1}{p t^{q-p}}\|u\|^{p} \\
& -\int\left(\frac{F(x, t u)}{(t u)^{q}} u^{q}-\frac{1}{q} \Gamma(x)|u|^{q}\right) d x
\end{aligned}
$$

$$
\longrightarrow-\infty \text {, }
$$

as $t \longrightarrow+\infty$. Thus, there exists $t>0$ such that $J(t u)<0$. 
Now, we define the Nehari manifold by

$$
\mathcal{N}:=\left\{u \in E \backslash\{0\}:\left\langle J^{\prime}(u), u\right\rangle=0\right\} .
$$

It is easy to prove that $\mathcal{N}$ is not empty. And we have the following lemma.

Lemma 4. Suppose that $(V),(\Gamma)$ and $\left(f_{1}\right)-\left(f_{4}\right)$ hold. Let $\theta \in$ $[0,1) \cup(1, \infty)$ and $u \in \mathcal{N}$; then $J(\theta u)<J(u)$.

Proof. By $\left(f_{4}\right)$, we have

$$
\begin{aligned}
f(x, s) & \leq \frac{f(x, t)}{|t|^{q-1}}|s|^{q-1}, \quad s<t \\
f(s) & \geq \frac{f(x, t)}{|t|^{q-1}}|s|^{q-1}, \quad s>t .
\end{aligned}
$$

Then

$$
\begin{array}{r}
F(x, t)-F(x, \theta t)=\int_{\theta t}^{t} f(x, s) d s \leq \frac{1-\theta^{q}}{q} f(x, t) t, \\
\forall \theta \geq 0, t \in \mathbb{R} .
\end{array}
$$

Let $h(\theta)=p \theta^{q}-q \theta^{p}$; then $h^{\prime}(\theta)=p q\left(\theta^{q-1}-\theta^{p-1}\right)$. By a simple calculation, we have $h^{\prime}(1)=0$ and $h(\theta)>h(1)=p-q$ for all $\theta \in[0,1) \cup(1, \infty)$. Thus,

$$
\frac{\theta^{p}-1}{p}-\frac{\theta^{q}-1}{q}=\frac{p-q-h(\theta)}{p q}<0 .
$$

Let $u \in \mathcal{N}$, it follows from (18) and (19) that

$J(\theta u)$

$$
\begin{aligned}
= & J(u)+\left(J(\theta u)-J(u)-\frac{\theta^{q}-1}{q}\left\langle J^{\prime}(u), u\right\rangle\right) \\
= & J(u)+\left[\frac{\theta^{p}-1}{p}-\frac{\theta^{q}-1}{q}\right]\|u\|^{p} \\
& +\int\left[\frac{\theta^{q}-1}{q} f(x, u) u-F(x, \theta u)+F(x, u)\right] d x
\end{aligned}
$$$$
<J(u) \text {. }
$$

Lemma 5. Suppose that $(V),(\Gamma)$ and $\left(f_{1}\right)-\left(f_{4}\right)$ hold; let $m:=$ $\inf _{\mathcal{N}} J$; then there exist $\left\{u_{n}\right\} \in E, c_{*} \in\left(\rho_{0}, m\right]$ satisfying

$$
\begin{aligned}
J\left(u_{n}\right) & \longrightarrow c_{*}, \\
\left(1+\left\|u_{n}\right\|\right)\left\|J^{\prime}\left(u_{n}\right)\right\| & \longrightarrow 0 .
\end{aligned}
$$

Proof. By (i) of Lemma 3, there exist $\delta_{0}>0$ and $\rho_{0}>0$ such that

$$
u \in E,\|u\|=\delta_{0} \Longrightarrow J(u) \geq \rho_{0} .
$$

Choose $v_{k} \in \mathscr{N}$ such that

$$
m \leq J\left(v_{k}\right)<m+\frac{1}{k}, \quad k \in \mathbb{N} .
$$

Since $J\left(t v_{k}\right)<0$ for large $t>0$, Mountain Pass Lemma implies that there exists $\left\{u_{k, n}\right\}_{n \in \mathbb{N}} \subset E$ satisfying

$$
\begin{aligned}
J\left(u_{k, n}\right) & \longrightarrow c_{k}, \\
\left\|J^{\prime}\left(u_{k, n}\right)\right\|\left(1+\left\|u_{k, n}\right\|\right) & \longrightarrow 0,
\end{aligned}
$$

\section{$k \in \mathbb{N}$,}

where $c_{k} \in\left[\rho_{0}, \sup _{t \geq 0} J\left(t v_{k}\right)\right]$. By Lemma 4 , we have $J\left(v_{k}\right)=$ $\sup _{t \geq 0} J\left(t v_{k}\right)$. Hence, by (23) and (24), we have

$$
\begin{aligned}
J\left(u_{k, n}\right) & \longrightarrow c_{k}<m+\frac{1}{k}, \\
\left\|J^{\prime}\left(u_{k, n}\right)\right\|\left(1+\left\|u_{k, n}\right\|\right) & \longrightarrow 0,
\end{aligned}
$$

\section{$k \in \mathbb{N}$.}

Now, we can choose a sequence $\left\{n_{k}\right\} \subset \mathbb{N}$ such that

$$
\begin{gathered}
J\left(u_{k, n_{k}}\right)<m+\frac{1}{k}, \\
\left\|J^{\prime}\left(u_{k, n_{k}}\right)\right\|\left(1+\left\|u_{k, n_{k}}\right\|\right)<\frac{1}{k}, \quad \\
k \in \mathbb{N} .
\end{gathered}
$$

Let $u_{k}=u_{k, n_{k}}, k \in \mathbb{N}$. Then, going if necessary to a subsequence, we have

$$
\begin{aligned}
J\left(u_{n}\right) & \longrightarrow c_{*} \in\left[\rho_{0}, m\right], \\
\left\|J^{\prime}\left(u_{n}\right)\right\|\left(1+\left\|u_{n}\right\|\right) & \longrightarrow 0 .
\end{aligned}
$$

\section{Proof of Theorem 1}

Proof of Theorem 1 . In view of Lemma 5, we find a Cerami sequence $\left\{u_{n}\right\}$ satisfying (21). By (18), we have

$$
\frac{1}{q} f\left(x, u_{n}\right) u_{n}-F\left(x, u_{n}\right) \geq 0 \text {, }
$$

for all $x \in \mathbb{R}^{N}, u_{n} \in E$.

Combining (21) and (28), for $n$ big enough, we have

$$
\begin{aligned}
c_{*}+1 \geq & J\left(u_{n}\right)-\frac{1}{q}\left\langle J^{\prime}\left(u_{n}\right), u_{n}\right\rangle \\
= & \left(\frac{1}{p}-\frac{1}{q}\right)\left\|u_{n}\right\|^{p} \\
& +\int\left[\frac{1}{q} f\left(x, u_{n}\right) u_{n}-F\left(x, u_{n}\right)\right] d x \\
\geq & \left(\frac{1}{p}-\frac{1}{q}\right)\left\|u_{n}\right\|^{p} .
\end{aligned}
$$


It follows that $\left\|u_{n}\right\|$ is bounded. Passing to a subsequence, we have $u_{n} \rightarrow u_{0}$ in $E$. By Lemma 2, we have $u_{n} \longrightarrow u_{0}$ in $L^{r}\left(\mathbb{R}^{N}\right)$ for $r \in\left[p, p_{\alpha}^{*}\right)$. Then, by (13) and the Hölder inequality, we have

$$
\begin{aligned}
& \left|\int\left(f\left(x, u_{n}\right)-f\left(x, u_{0}\right)\right)\left(u_{n}-u_{0}\right) d x\right| \\
& \leq \varepsilon \int\left(\left|u_{n}\right|^{p-1}+\left|u_{0}\right|^{p-1}\right)\left|u_{n}-u_{0}\right| d x \\
& \quad+C_{\varepsilon} \int\left(\left|u_{n}\right|^{r-1}+\left|u_{0}\right|^{r-1}\right)\left|u_{n}-u_{0}\right| d x \\
& \leq \varepsilon\left(\left\|u_{n}\right\|_{p}^{p-1}+\left\|u_{0}\right\|_{p}^{p-1}\right)\left\|u_{n}-u_{0}\right\|_{p} \\
& \quad+C_{\varepsilon}\left(\left\|u_{n}\right\|_{r}^{r-1}+\left\|u_{0}\right\|_{r}^{r-1}\right)\left\|u_{n}-u_{0}\right\|_{r} \rightarrow 0
\end{aligned}
$$

and

$$
\begin{aligned}
& \left|\int \Gamma(x)\left(\left|u_{n}\right|^{q-2} u_{n}-\left|u_{0}\right|^{q-2} u_{0}\right)\left(u_{n}-u_{0}\right) d x\right| \\
& \quad \leq\|\Gamma\|_{\infty} \int\left(\left|u_{n}\right|^{q-1}+\left|u_{0}\right|^{q-1}\right)\left|u_{n}-u_{0}\right| d x \\
& \quad \leq\left(\left\|u_{n}\right\|_{q}^{q-1}+\left\|u_{0}\right\|_{q}^{q-1}\right)\left\|u_{n}-u_{0}\right\|_{q} \longrightarrow 0 .
\end{aligned}
$$

It follows from (30), (31) and Simon inequality $\left(\left(|a|^{p-2} a-\right.\right.$ $\left.\left.|b|^{p-2} b\right)(a-b) \geq\left(1 / 2^{p-2}\right)|a-b|^{p}\right)$ that

$$
\begin{aligned}
& \left\langle J^{\prime}\left(u_{n}\right)-J^{\prime}\left(u_{0}\right), u_{n}-u_{0}\right\rangle \\
& =\int \frac{1}{|x-y|^{N+\alpha p}}\left[\left|u_{n}(x)-u_{n}(y)\right|^{p-2}\right. \\
& \cdot\left(u_{n}(x)-u_{n}(y)\right)-\left|u_{0}(x)-u_{0}(y)\right|^{p-2} \\
& \left.\cdot\left(u_{0}(x)-u_{0}(y)\right)\right]\left[u_{n}(x)-u_{n}(y)-u_{0}(x)\right. \\
& \left.+u_{0}(y)\right] d x d y+\int V(x)\left[\left|u_{n}\right|^{p-2} u_{n}\right. \\
& \left.-\left|u_{0}\right|^{p-2} u\right]\left(u_{n}-u_{0}\right) d x-\int\left(f\left(x, u_{n}\right)\right. \\
& \left.-f\left(x, u_{0}\right)\right)\left(u_{n}-u_{0}\right) d x+\int \Gamma(x)\left(\left|u_{n}\right|^{q-2} u_{n}\right. \\
& \left.-\left|u_{0}\right|^{q-2} u_{0}\right)\left(u_{n}-u_{0}\right) d x \geq \frac{1}{2^{p-2}} \\
& +\int \frac{\left|\left(u_{n}(x)-u_{n}(y)\right)-\left(u_{0}(x)-u_{0}(y)\right)\right|^{p}}{|x-y|^{N+\alpha p}} d x d y \\
& +\frac{1}{2^{p-2}} \int V(x)\left|u_{n}-u_{0}\right|^{p} d x+o_{n}(1)=\frac{1}{2^{p-2}} \| u_{n} \\
& -u_{0} \|^{p}+o_{n}(1) .
\end{aligned}
$$

On the other hand, by $\left\langle J^{\prime}\left(u_{n}\right), u_{n}-u_{0}\right\rangle \longrightarrow 0$ and $u_{n} \rightarrow u_{0}$, we have

$$
\left\langle J^{\prime}\left(u_{n}\right)-J^{\prime}\left(u_{0}\right), u_{n}-u_{0}\right\rangle \longrightarrow 0 .
$$

Combining (32) and (33), we have $u_{n} \longrightarrow u_{0}$ in $E$. Then, by $J \in C^{1}(E, \mathbb{R})$, we have $J^{\prime}\left(u_{0}\right)=0$. By (28), Lemma 5 , and Fatou's lemma, we have

$$
\begin{aligned}
m \geq & c_{*}=\lim _{n \rightarrow \infty}\left[J\left(u_{n}\right)-\frac{1}{q}\left\langle J^{\prime}\left(u_{n}\right), u_{n}\right\rangle\right] \\
= & \left(\frac{1}{p}-\frac{1}{q}\right) \lim _{n \rightarrow \infty}\left\|u_{n}\right\|^{p} \\
& +\lim _{n \longrightarrow \infty} \int\left[\frac{1}{q} f\left(x, u_{n}\right) u_{n}-F\left(x, u_{n}\right)\right] d x \\
\geq & \left(\frac{1}{p}-\frac{1}{q}\right)\left\|u_{0}\right\|^{p} \\
& +\int\left[\frac{1}{q} f\left(x, u_{0}\right) u_{0}-F\left(x, u_{0}\right)\right] d x \\
= & J\left(u_{0}\right)-\frac{1}{q}\left\langle J^{\prime}\left(u_{0}\right), u_{0}\right\rangle=J\left(u_{0}\right) .
\end{aligned}
$$

This shows that $J\left(u_{0}\right) \leq m$ and so $J\left(u_{0}\right)=m=\inf _{\mathcal{N}} J>$ 0 .

\section{Data Availability}

No data were used to support this study.

\section{Conflicts of Interest}

The authors declare that they have no conflicts of interest.

\section{Authors' Contributions}

All authors contributed equally and significantly in writing this article. All authors read and approved the final manuscript.

\section{Acknowledgments}

This work is supported by the Hainan Natural Science Foundation (Grant nos. 118MS002 and 117005), National Natural Science Foundation of China (Grant nos. 11461016 and 11571370), China Postdoctoral Science Foundation Funded Project (Grant no. 2017M612577), and Young Foundation of Hainan University (Grant no. hdkyxj201718).

\section{References}

[1] D. Applebaum, "Lévy processes-from probability to finance and quantum groups," Notices of the American Mathematical Society, vol. 51, no. 11, pp. 1336-1347, 2004.

[2] N. Laskin, "Fractional quantum mechanics and Lévy path integrals," Physics Letters A, vol. 268, no. 4-6, pp. 298-305, 2000.

[3] R. Metzler and J. Klafter, "The restaurant at the end of the random walk: recent developments in the description of anomalous transport by fractional dynamics," Journal of Physics A: Mathematical and General, vol. 37, no. 31, pp. R161-R208, 2004 . 
[4] B. Bieganowski, "Solutions of the fractional Schrödinger equation with a sign-changing nonlinearity," Journal of Mathematical Analysis and Applications, vol. 450, no. 1, pp. 461-479, 2017.

[5] A. Szulkin and T. Weth, "Ground state solutions for some indefinite variational problems," Journal of Functional Analysis, vol. 257, no. 12, pp. 3802-3822, 2009.

[6] X. H. Tang, "Non-Nehari manifold method for superlinear Schrödinger equation," Taiwanese Journal of Mathematics, vol. 18, no. 6, pp. 1957-1979, 2014.

[7] X. H. Tang, "Non-Nehari manifold method for asymptotically periodic Schrödinger equations," Science China Mathematics, vol. 58, no. 4, pp. 715-728, 2015.

[8] E. Di Nezza, G. Palatucci, and E. Valdinoci, "Hitchhiker's guide to the fractional Sobolev spaces," Bulletin des Sciences Mathématiques, vol. 136, no. 5, pp. 521-573, 2012.

[9] V. Ambrosio, "Multiple solutions for a fractional p-Laplacian equation with sign-changing potential," Electronic Journal of Differential Equations, vol. 2016, article 151, 12 pages, 2016. 


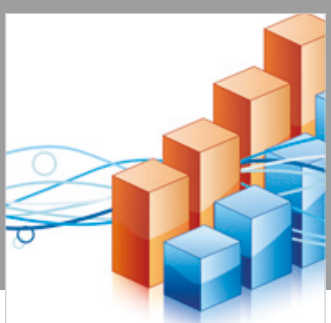

Advances in

Operations Research

\section{-n-m}
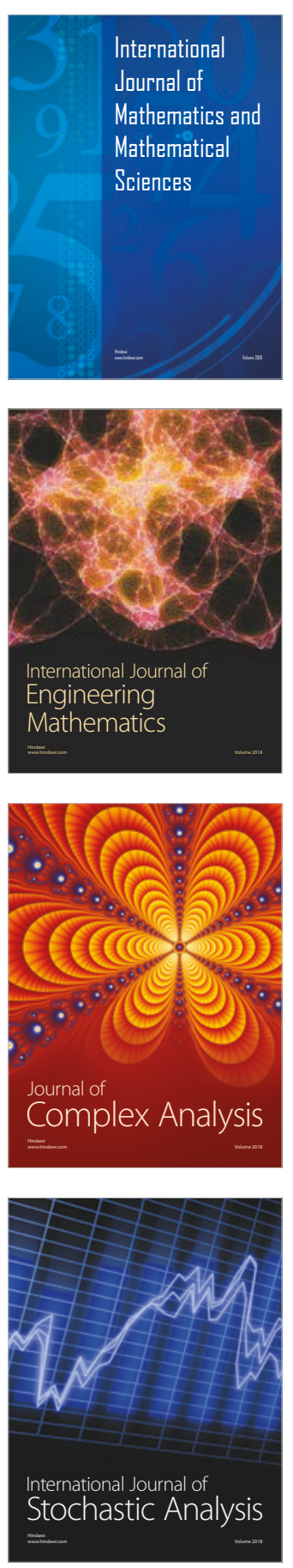
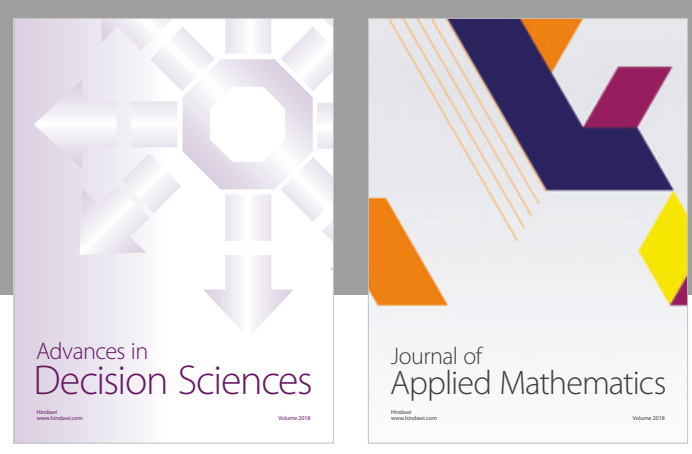

Journal of

Applied Mathematics
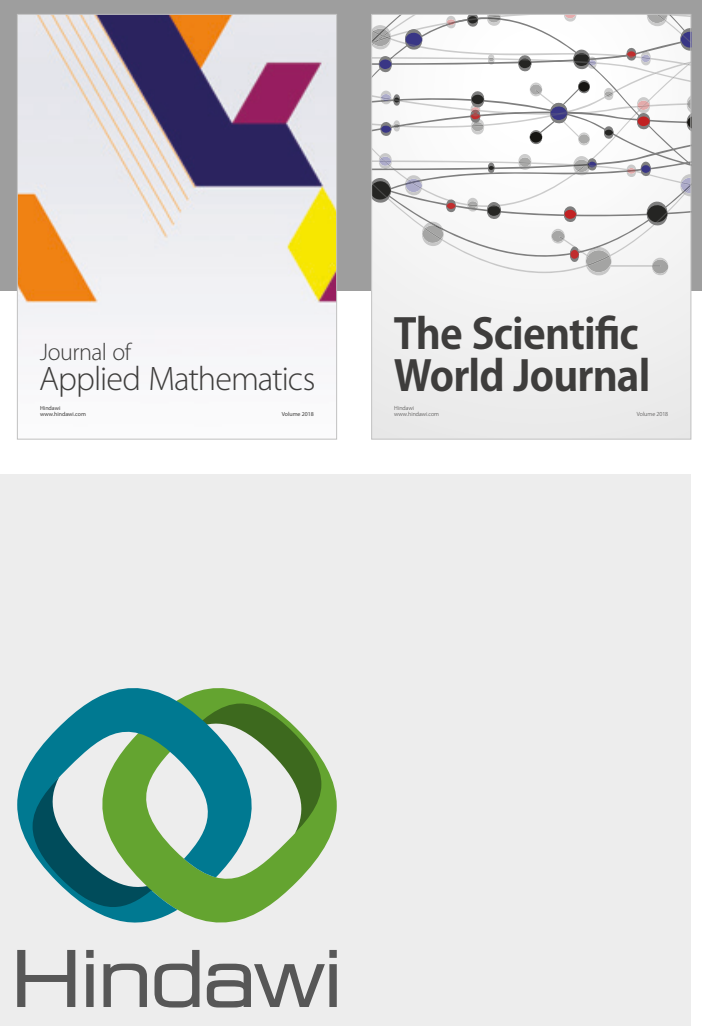

Submit your manuscripts at

www.hindawi.com

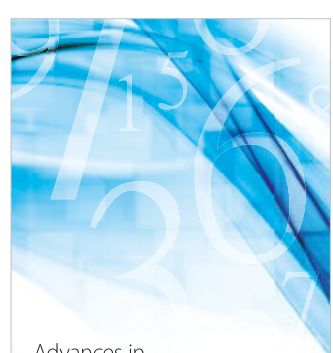

Advances in
Numerical Analysis
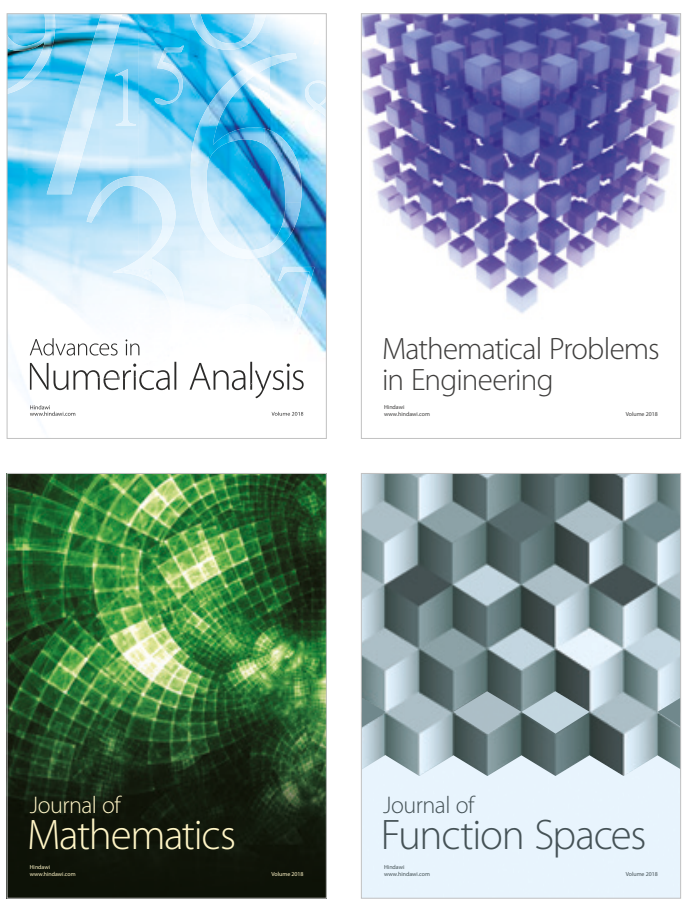

Mathematical Problems in Engineering

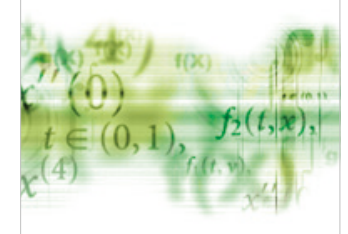

International Journal of

Differential Equations

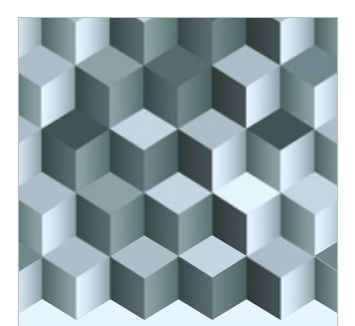

Journal of

Function Spaces

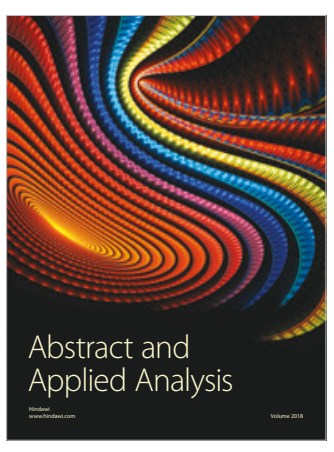

The Scientific

World Journal

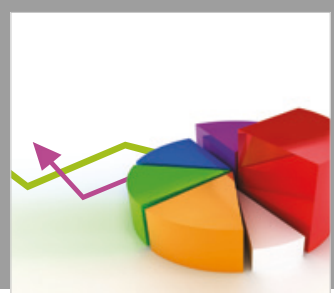

Journal of

Probability and Statistics
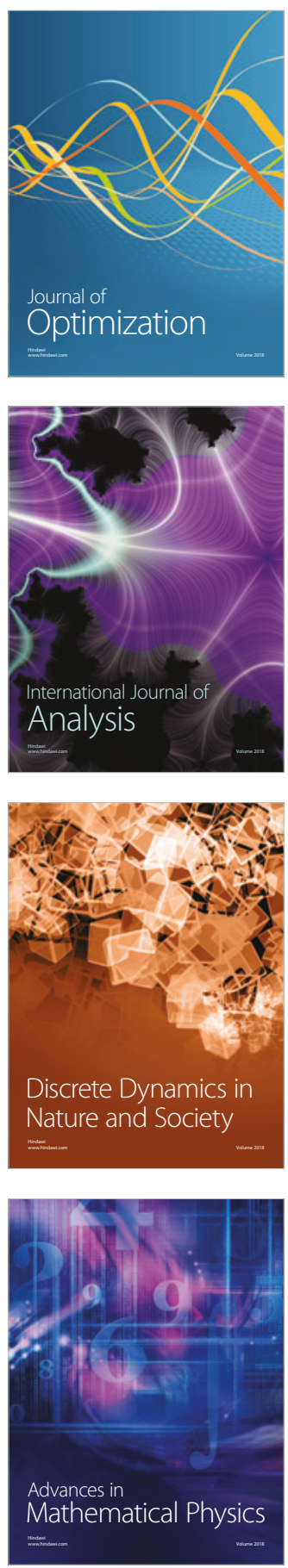